\title{
Tecnologías aplicadas a la seguridad ciudadana: desafíos para la justicia transicional ante nuevos mecanismos de control social
}

\section{Technologies for citizen security: challenges for transitional justice to new mechanisms of social control}

ARTÍCULO

\author{
Milton Lechner \\ Universidad Nacional de Lanús y Universidad Nacional Arturo Jauretche, Argentina. \\ mlechner@unla.edu.ar
}

Recibido: abril del 2016

Aceptado: agosto del 2016

\section{Resumen}

El presente artículo pretende brindar una reflexión sobre las políticas públicas de seguridad ciudadana y las fuerzas de seguridad públicas, que han incorporado un conjunto de costosas y sofisticadas innovaciones tecnológicas, con el fin de mejorar la eficacia sobre la prevención y reducción del delito. Por ello, entendemos que la justicia transicional es un actor fundamental para estas políticas, debido a que si no son reguladas y auditadas, pueden provocar una sistemática vulneración de derechos, conformando un sistema de control social generalizado por parte de los Estados.

Palabras claves: tecnologías; seguridad ciudadana; seguridad electrónica; red panóptica; control social; justicia transicional.

\section{Abstract}

This article aims to provide a reflection on public policies of public safety and public security forces, who have built a set of costly and sophisticated technological innovations in order to improve efficiency in preventing and reducing crime. Therefore, we understand that transitional justice is a fundamental actor for these policies, because if they are not regulated and audited, may cause a systematic violation, forming a widespread system of social control by the States.

Keywords: technology; citizen security; electronic security; panoptic network; social control; transitional justice.

\section{Introducción}

Este trabajo nace del interés por investigar y analizar los procesos teóricos que describen las tecnologías empleadas actualmente dentro de las políticas de prevención y 
punición del delito por parte del Estado. De tal forma se plantea un abordaje de las problemáticas que se presentan a la hora de implementar las tecnologías dentro de la perspectiva de la inclusión social y el resguardo de los derechos humanos.

El artículo también está inspirado por un conjunto de autores tales como Foucault, Garland, Crawford, Orwell y Huxley, entre otros. Autores que presentaron en sus obras un Estado totalitario, centrado en el control de las personas y la sociedad en su conjunto, tendiente a generar un vínculo de poder hegemónico, estableciendo el binomio = dominado (individuo-ciudadano) y dominador (FFSS - Estado).

La problemática de la (in)seguridad, en los últimos años, ha sido un tema prioritario en la agenda de los Estados; tanto de la órbita nacional como provincial y municipal. Para ello, se generaron diversas políticas públicas dentro de cada una de sus jurisdicciones con el motivo de lograr el bienestar social. Como veremos estas políticas relacionadas a la prevención situacional, pueden afectar también las normas de convivencia y los derechos de los ciudadanos.

El objetivo principal del trabajo se concentró en el análisis de la tecnificación de la seguridad ciudadana a través del uso de las nuevas tecnologías y su relación con las políticas públicas que, como veremos, muchos Estados utilizaron para el control social. Esto generó nuevos parámetros para la justicia transicional la cual tendrá que adaptarse a los nuevos paradigmas tecnológicos.

Se comenzó por analizar la forma en la que el Estado tecnificaba y en mostrar cuáles eran las necesidades sociales que daban origen a este fenómeno. Se entiende que actualmente la tecnología es un elemento primordial al formular y desarrollar políticas públicas relacionadas con la problemática de la inseguridad y la justicia.

Seguidamente, se describe cómo fue la evolución de las TIC's dentro del campo de la seguridad ciudadana y cuál fue el origen de su aplicación durante las sociedades disciplinarias. A su vez, nos detendremos en los principales marcos teóricos de la criminología basados en las tecnologías como aporte para la prevención y punición del delito.

Para ello, se comienza a trabajar desde el paradigma del orden, desde el cual las sociedades eran sistematizadas a partir de las políticas de represión y punición de la criminalidad. Luego arribaremos al paradigma de la nueva prevención donde se emplean otras estrategias para abordar las conflictividades que derivan en el delito y la criminalidad. Por su parte, se identifican cuáles son las nuevas tecnologías que conforman los principales sistemas de seguridad electrónica, que fueron incorporadas al campo de la seguridad ciudadana. En tal sentido, intentaremos determinar a qué ámbito de aplicación pertenecen estas nuevas tecnologías y cómo se fueron adaptando a la sociedad dentro del campo de la seguridad. Se indaga en torno a la existencia de una posible relación entre las nuevas formas de control social, los intereses políticos, militares y la anulación de 
poder de diversos grupos de la sociedad, generando variadas situaciones relacionadas al espionaje implícito. Siempre utilizando los avances tecnológicos para perpetuar el control y dominación de la sociedad.

Por último, se detecta cómo estas políticas públicas basadas en la aplicación de la tecnología sobre la seguridad son instrumentadas para vigilar desde otras partes del hemisferio a las sociedades y sus Estados, avasallando así un conjunto de normas internas y Tratados Internacionales relacionados con los Derechos Humanos.

A lo largo de este trabajo se busca demostrar la hipótesis que establece que las tecnologías aplicadas a la seguridad ciudadana se han constituido en una herramienta fundamental del Estado para ejercer nuevos métodos de control social.

\section{La seguridad ciudadana y las tecnologías}

Las transformaciones de las sociedades en estos últimos 20 años, han modificado la naturaleza del delito, en tanto que hoy las tecnologías modifican las fronteras y los campos de acción. Debido a ello, los conceptos de seguridad se han reformulado, como se han modificado las formas de vida de los ciudadanos, de igual modo las instituciones han cambiado su mirada con respecto a la problemática delictual. Hoy en día podemos ver que se tiene en cuenta la participación ciudadana y a la comunidad al momento de formular políticas de seguridad, esto se lo podemos adjudicar, entre otras cosas, a los nuevos modos de abordar la problemática y entender la seguridad, desde la función de la justicia transicional en Latinoamérica.

Pero veremos cómo el desarrollo de nuevas tecnologías de la comunicación y la información, de ahora en más TIC's, hicieron que los avances tecnológicos se fueran aplicando paulatinamente como una herramienta para la resolución de los problemas y las demandas de seguridad en las poblaciones; con el objetivo de garantizar la prevención, la paz y una mejor calidad de vida de sus habitantes.

Las TIC's se convirtieron en un actor fundamental al momento de formular y desarrollar políticas públicas relacionadas a las problemáticas de la inseguridad, con el motivo de atenuar los niveles delictuales. De esta manera, vemos que los Estados se tecnificaron con métodos y diseños tanto de prevención como de punición contra los delitos que ocurridos dentro de sus jurisdicciones.

Para comprender un poco más en profundidad el proceso de tecnificación del Estado dentro del campo de la seguridad ciudadana, se debió tener presente que en términos generales, la noción de seguridad estaba vinculada a un valor, a un objetivo deseable aunque ciertamente inasible- apuntado a la incertidumbre, la desprotección, la inseguridad, las amenazas. Esta reducción o disminución de la incertidumbre es inasible en tanto 
aquello que atenta contra las certezas es cambiante y está históricamente determinado. (Rangugni y Russo, 2010)

De tal forma, las TIC's se complementaban como herramientas para generar una reducción de la incertidumbre y el riesgo social, como una protección ante las amenazas. Esas certezas (Información/conocimiento) que generaban para dar confianza y protección, tenían que estar en constante desarrollo y actualización, en concordancia con las mutaciones de los delitos y futuras amenazas.

En la misma línea, se comprendió la noción de Seguridad Ciudadana al hacer énfasis en la participación de la ciudadanía como actor de derecho y productor de seguridad. La misma se definió como

...el derecho de los integrantes de la sociedad de desenvolverse cotidianamente con el menor nivel posible de amenazas a su integridad personal, sus derechos y el goce de sus bienes. Se asienta en la obligación del Estado de satisfacer las necesidades del ciudadano, a cambio de la delegación de poder que este ha hecho en los responsables de conducir el aparato estatal. (González, 2003, p.16)

Este concepto supera a la idea de seguridad pública y puede verse en contraposición a las Doctrinas de Seguridad Nacional implementadas décadas pasadas en Latinoamérica, ligada al mantenimiento del orden público por parte de un Estado militarizado.

\section{La seguridad desde la perspectiva tecnológica}

El uso de las nuevas tecnologías en las últimas décadas ha ido en aumento de manera sustancial y esto ha significado un cambio en los hábitos sociales, especialmente comerciales, financieros y comunicativos. La implementación de las formas de pago electrónicos, conjuntamente a la proliferación de cajeros automáticos, el e-mail, los teléfonos celulares, los GPS, el comercio electrónico, etc. han sido prueba de ello, con la gran red de Internet que interconecta a las TIC's.

Muchos de estos dispositivos, fueron creados en primera instancia con fines militares. Luego se fueron acondicionando a la sociedad civil con un objetivo comercial. Mattelart explicó que la Agencia de Seguridad Nacional (NSA) del gobierno de Estados Unidos, fue la creadora de lo que hoy conocemos como Internet:

Una de sus misiones era agrupar a los actores de la innovación técnica contra el enemigo catalogado como "global", el comunismo mundial. Así se edificó el complejo militaro-industrial, fruto de la sinergia entre la investigación científica, la industria y los organismos de inteligencia militar o civil. Es en ese marco de cooperación que fue inventado, en 1958, Arpanet, antepasado de Internet. El organismo que fue el eje giratorio, la DARPA (Defense Advanced Research Projects Agency), es hoy el epicentro del proyecto de integración de las redes y bancos de datos. (Mattelart, 2007, p. 7) 
Los dispositivos generados por la industria de la seguridad electrónica fueron los sistemas de intrusión, de videovigilancia, de controles de accesos, de reconocimientos biométricos, de geolocalización, de comunicación y de gestión de central de alarmas (López Martínez, 2013).

Ahora, pasaremos a ver detalladamente, qué características tuvieron los principales sistemas de seguridad y cuáles fueron sus funciones específicas, como así también su utilización como dispositivos de control dentro del campo de la seguridad.

\section{- Sistemas de Geolocalización}

El sistema de geolocalización se basa en el sistema conocido mundialmente como GPS (Global Positioning System) o Sistema de Posicionamiento Global. Está compuesto por una red de 24 satélites denominada NAYSTAR, situados en una órbita a unos 20.200 kilómetros de la Tierra, en tanto que los receptores GPS permiten determinar cualquier posición en cualquier lugar del planeta y su respectiva altitud, durante las 24 hs. y bajo cualquier condición meteorológica. A través de la triangulación logra la altitud, latitud y longitud de los objetos localizados en mapas digitalizados.

La expansión del GPS está particularmente ligada a los nuevos sistemas operativos como Android que va desde el uso del celular para la localización punto a punto, hasta las principales aplicaciones Web como Twitter, Google+ y Facebook, las cuales publican la ubicación del dispositivo automáticamente.

Los sistemas se aplicaron a la seguridad pública y se comenzó a incorporar paulatinamente este tipo de tecnología en los patrulleros de las fuerzas de seguridad, con el objetivo de controlar el accionar y sus recorridos. En ese sentido, se observó cómo la geolocalización pasó a constituirse en un dispositivo disciplinario y de control, individualizando tanto a los ciudadanos, como también al mismo Estado y a sus agentes de seguridad.

- Sistemas de videovigilancia públicos y privados

Los sistemas de videovigilancia se constituyeron en una forma de vigilancia sobre la totalidad de sus ciudadanos y sus instituciones alcanzándose así, niveles de control social nunca antes observados.

$\mathrm{Si}$ bien los sistemas de videovigilancia son utilizados dentro de las políticas de prevención, con el fin de tener un objetivo disuasorio, en general pasan a ser un elemento represivo, debido a que resguardan la información por un tiempo determinado; para luego, ante la ocurrencia de un hecho delictivo o su sospecha, procede a buscar en los registros de imágenes para individualizar a los sospechosos del resto de la sociedad. Los dispositivos de control se mecanizan a través del poder como información y la vigilancia 
como seguridad. Así lo expresa Capistrán Bañuelos (2003) cuando afirma que “...Todo ello expresado en la imagen no sólo como soporte y representación, sino como sustitución de la realidad, imagen como instante puro o presente puro, imagen como prueba judicial, imagen como espectáculo de lo real".

Los sistemas de videovigilancia se fueron innovando a sí mismos y pasaron a modificar sus estructuras como sus prestaciones, de a poco se fueron instalando en diferentes dispositivos móviles, entre ellos los drones y patrulleros tecnológicos de las distintas fuerzas de seguridad, y junto a software diseñados especialmente para las fuerzas que poseen múltiples aplicaciones.

Estos sistemas, al igual que los fijos, poseen programas de reconocimiento facial de las personas como también programas de identificación de patentes. Ambos enlazados online a las base de datos que están permanentemente actualizados y cotejados con los datos obtenidos por las unidades móviles, todo conectado automáticamente a través de las imágenes en tiempo real con la central de monitoreo, la cual activará en forma también automática una alerta para que la unidad más próxima acuda a la ubicación de ese móvil policial en caso de necesitarlo, como también vimos en el caso de los GPS.

Por su parte, los patrulleros tecnológicos están equipados por un moderno sistema de vigilancia que tiene 8 cámaras: 6 en el puente del techo que cubren 360 grados alrededor del auto. 1 domo también ubicado en el techo, controlado desde el auto con una pantalla interactiva (touchscreen) o de forma remota desde el Centro de Monitoreo.1 cámara fija en el interior del auto que también graba audio. (Ministerio de Seguridad de la Nación Argentina, 2011)

También existe otro dispositivo más novedoso para acceder a lugares desde el aire, que son los Drones. Quienes están en desarrollo continuo y son diseñados para diferentes aplicaciones, entre otras, la seguridad ciudadana. En ese sentido, lo que no se podía vigilar por tierra, se comenzó a vigilar por aire, a bajo costo y con pocos recursos humanos.

Las imágenes captadas por las redes de cámaras del Estado como también desde los dispositivos privados, dan forma a una mega red de vigilancia estilo panóptico que se extiende diariamente con gran impulso en las sociedades modernas, donde no hay un control sobre el que vigila y sobre qué se vigila, donde el ciudadano es convertido en un vigilante de sí mismo dentro del sistemas de videovigilancia tipo red, y no está libre del riesgo de convertirse en objeto de vigilancia, donde todos tienen la capacidad vigilar a todos, registrando sin control imágenes de otros ciudadanos.

Igualmente, todos estos sistemas de videovigilancia se van a complementar con la biometría, para conformar un sistema combinado de identificación más eficaz y eficiente de las personas. 
- Sistemas biométricos y de controles públicos

Siguiendo lo expresado en relación a los sistemas de geolocalización como los de videovigilancia, pasamos ahora a las tecnologías biométricas, último eslabón en la complementación del sistema de identificación y control.

Se entiende por reconocimiento biométrico a los métodos automatizados que aseguran el reconocimiento de individuos con base en rasgos físicos o conductuales distinguibles. Las tecnologías que se usan en biometría incluyen el reconocimiento de huellas dactilares, de rostros, de patrones de las venas, del iris, de voz y del tecleo, entre otros. (Thill, 2011, p. 28)

Resulta obvia la necesidad de mantener entornos públicos y privados controlados para preservar la seguridad, bien se trate de personas, objetos o información. No obstante, la condición de control a través de la vigilancia no puede ser absoluta y se requieren de sistemas sofisticados para los controles públicos. La solución para este tipo de seguridad es la implantación de los sistemas biométricos, que desde la creación de la dactiloscopia por Juan Vucetich, se vienen desarrollando y aplicando para la identificación de las personas como para dar acceso a diferentes lugares restringidos y específicos para cada actividad deseada.

Los sistemas biométricos son sistemas informáticos de reconocimiento con base en uno o varios patrones biológicos. Estos requieren datos biométricos a un individuo, extraen un patrón de estos datos adquiridos y comparan el ejemplo contra una plantilla previamente registrada. Ésta, de acuerdo al tipo de aplicación, puede estar almacenada en una base de datos centralizada. Uno de los sistemas más conocidos que disponen las fuerzas de seguridad, es el sistema AFIS que cuenta con la capacidad para identificar en forma precisa a una persona a través de la huella digital, su aplicación es tanto para medidas de orden preventivo como represivo.

En relación, todas estas innovaciones tecnológicas, no siempre son entendidas como una implementación positiva sobre la prevención del delito. El criminólogo Adam Crawford critica claramente la táctica de prevención situacional y su uso desmedido de las TIC's, sosteniendo que:

Puede promover una fe ciega en la tecnología que puede ser injustificada y que está guiada fundamentalmente por intereses comerciales de la creciente industria de la seguridad (alarmas, CCTV, iluminación, etc.) y desplaza el potencial de la importancia de la agencia humana en las actividades de control del crimen (Crawford, 1998, p.112)

\section{El nacimiento de las sociedades panópticas}

La sociedad disciplinaria, como la llamó Michel Foucault, se basadaba en la obra "EI Panóptico" de Jeremy Bentham. Foucault realizó un estudio de una serie de procesos que trasformaron a la sociedad punitiva del siglo XVIII, en la sociedad disciplinaria del siglo 
XIX. De tal forma, se dedicó al estudio de los dispositivos disciplinarios desde la perspectiva de la cárcel y utilizó el término "disciplina" como el conjunto de técnicas y procedimientos con los cuales se buscó producir cuerpos políticamente dóciles y económicamente rentables. (Foucault, 2002)

...la 'disciplina' no puede identificarse ni con una institución ni con un aparato; es un tipo de poder, una modalidad para ejercerlo, que comporta a todo un conjunto de instrumentos, de técnicas, de procedimientos, niveles de aplicación, de objetivos; es una 'física' o una 'anatomía' del poder, una tecnología. (Foucault, 2002, p. 218)

No sólo la cárcel estuvo atravesada por estos procesos, sino también la red de instituciones que conformaban a la sociedad del siglo XIX, como las fábricas, las comisarías, los hospitales y las escuelas, donde a través de los mecanismos de la sociedad disciplinaria, se generaba la nueva tecnología del poder.

Las instituciones disciplinarias produjeron una maquinaria de control que ha funcionado como un microscopio de la conducta; las divisiones tenues y analíticas han llegado a formar, en torno de los hombres, un aparato de observación, de registro y de encauzamiento de la conducta. (Foucault, 2002)

El Panóptico está conformado por las siguientes características que define el principio general de esta nueva dinámica de poder de las sociedades de normalización disciplinaria que podrían considerarse como una invención tecnológica equivalente a la máquina de vapor en el proceso productivo. $Y$ se la describe como una estructura arquitectónica que posee una torre central (donde se realizan las tareas de vigilancia y control de los individuos) circundada por un anillo constituido por celdas, que son habitadas por los detenidos. (Foucault, 1994)

El panóptico se diseñó para optimizar el tiempo de la visualización de los detenidos. Los que ocupaban las celdas eran vistos, pero ellos no podían ver a quienes los observaban desde la torre central. La acción del modelo era poder vigilar sin ser vistos, resultando eficaz y financieramente económica, su ejecución.

El individuo era sometido a la vigilancia, no podía ver si era realmente vigilado y no tenía certeza de la acción de vigilancia; pero sí podía ser consciente de la posibilidad de estar siendo vigilado. De esta manera, la acción de la vigilancia pasaba a modificar la conducta de los detenidos, convirtiendo a cada individuo en el normalizador de sí mismo. (Castro, 2014)

Consecuentemente, estas tecnologías de poder cobran importancia por sus mecanismos de observación que son capaces de controlar y modificar en el comportamiento de los individuos, estableciendo sus tareas, sus conductas, como legitimando el orden y el control social. (García Jiménez, 2009)

\section{La sociedad del orden}


Los mecanismos disciplinarios conforman las tecnologías de poder que se focalizan en el orden de los individuos; donde el panóptico es un ordenador de cuerpos y la sociedad sería donde cada individuo se sitúa en el lugar que le corresponde socialmente, generando así un ordenamiento para un adecuado control social. (Nievas, 1999)

De esta manera, los dispositivos de seguridad conforman al territorio y su soberanía, utilizando a la estadística como solución para los problemas demográficos y aplican el sistema jurídico simultáneamente con la disciplina -a través de cálculos y tácticas en un conjunto de instituciones (policías, escuelas, juzgados, etc.)- con el objetivo del gobierno de la población, para obtener el control y el ordenamiento necesario, estableciendo una estructura de poder elemental del Estado. No olvidemos que el poder "...tiene como blanco principal la población, por forma mayor de saber la economía política y por instrumento técnico esencial los dispositivos de seguridad." (Nievas, 1999, p. 81)

Así, el poder del Estado se fortaleció a partir de dos nuevas estructuras desarrolladas en el ámbito diplomático-militar que se ocupó de la política exterior y la Policía para la política interior. La Policía tuvo como objetivo la reglamentación de la vida de los ciudadanos. Por ende, las sociedades se fueron conformando dentro de un paradigma del orden, en el cual el orden racional del individuo se establecía dentro de los parámetros de lo normal y lo normado. También la sociedad generaba métodos para asegurar que sus miembros respondieran a un orden social dominante y hegemónico, diferenciando los comportamientos para definirlos como problemáticos, peligrosos, enfermos o desviados; desconociendo las conflictividades y problemas sociales.

En este sentido, los conflictos eran entendidos como un desorden y por esto, era necesario restablecer el control social de alguna manera. En la misma línea de pensamiento, el fenómeno delictual era entendido como un grave desorden o una desviación del equilibrio social. Según esta visión, toda conflictividad era sinónimo de "desorden" y como tal un desequilibrio de la armonía social que debía ser reordenada o restablecida. La idea central de este paradigma del orden provino del desarrollo del pensamiento político occidental; se trataba del orden natural propio del pensamiento grecorromano, el molde de una sociedad ordenada y estamental que ha calado muy hondo en nuestra comprensión de los fenómenos sociales. (Binder, 2009)

En consecuencia, las políticas de seguridad se proponen implantar el orden social, en sintonía con las teorías económicas postuladas desde el mercantilismo y seguidamente, por el liberalismo. Las políticas de seguridad fundaron mayormente una visión simplificadora, esquemática y de forma directa al conjunto de parámetros del orden, enmarcados dentro de conceptos tradicionales del orden público, orden interior e incluso seguridad interior. Así se conformaron las instituciones de seguridad desde hace más de 200 años hasta la actualidad. (Binder, 2009) 
La represión del delito fue una de las herramientas más utilizadas para instaurar en forma violenta el orden disoluto. Entendiéndola como, una acción en la cual se interviene posteriormente, después que el delito ha sido causado, para luego castigar al sujeto por lo realizado y disciplinar a la sociedad como espectadora del conflicto.

Esto conllevó a grandes cuestionamientos a este paradigma y sus políticas de seguridad, donde las políticas de "mano dura" denominada demagogia punitiva, generaron un malestar creciente en la sociedad y tensiones en relación con el Estado, acrecentando la violencia y a su vez, sin poder solucionar la ocurrencia del delito.

Estas políticas fueron denominadas de "etiquetamiento social" y de una escalada violenta contra el delito, implementadas por las instituciones encargadas de velar por la seguridad pública. (CELS y otros, 2003)

De este modo, todos los controles de estas políticas giran en torno a los delitos contra la propiedad privada, donde se estigmatiza una gran parte de la sociedad por su poder adquisitivo y/o lugares de residencia, como por ejemplo: ciudadanos de bajos recursos provenientes de barrios populares, asentamientos y/o villas de emergencia.

\section{Del paradigma del "Orden" a la "Nueva Prevención"}

A partir de la década de 1970, los modelos de las instituciones dedicadas a la seguridad pública sufrieron grandes transformaciones en los países más desarrollados. Estas modificaciones sociales y políticas se empezaron a implementar en países del tercer mundo a partir de la década del ' 90 . Es importante observar cuál es el contexto en el que se crea la Nueva Prevención, teniendo en cuenta que en primera instancia, los países estaban en crisis con el modelo anterior, observando que las tasas criminales ascendían y no podían continuar con las políticas basadas en la demagogia punitiva. Por lo cual, se vieron obligados a implementar otras políticas públicas de seguridad, acompañadas de un conjunto de transformaciones sociales, políticas y culturales. (Sozzo, 2008)

Las características fundamentales de la Nueva Prevención se refieren a la creciente delegación de funciones en materia de seguridad -antes centralizadas en organismos públicos dedicados a la seguridad, donde diferentes instituciones que componen los gobiernos locales, el sistema judicial y organizaciones civiles comienzan a participar activamente en el desarrollo y toma de decisiones concretas destinadas a la prevención delictual. Por su parte, la comunidad adoptó también la responsabilidad de ser un actor fundamental en la prevención y pasó a ser una fuente importante de información, por lo cual la participación ciudadana comenzó a ser un instrumento para diseñar estrategias en conjunto con las instituciones de seguridad. (Pat O'Malley, 2006)

Estas medidas fueron enfocadas con el objetivo primordial en la prevención del delito. Se actuaba antes que se origine el delito y se entendía que el accionar de las 
fuerzas de seguridad serían más eficaces a la hora de su intervención. La nueva prevención tuvo varias consideraciones sobre la represión del delito, pero se centró en las etapas pre-delictuales, operando sobre tres ejes fundamentales: la primera fue la actuación anticipada en situaciones favorables a la ocurrencia de hechos violentos y delictivos; la segunda, la asistencia a sectores y grupos sociales que estaban involucrados en situaciones conflictivas entre sí como con la ley; y por último, el desarrollo de acciones y actividades que se sustentaban en la prevención delictual. (Varela y Arriola, 2010)

El primer punto puso énfasis en modificar situaciones que posibilitaban la ocurrencia de conflictos violentos y acciones delictivas. Este tipo de prevención se la denominó Prevención Situacional. La misma se caracterizó por analizar los acontecimientos y circunstancias que eran favorables para la comisión de actos violentos y delictivos, con el motivo de diseñar y determinar medidas que redujeran la posibilidad de concertar los delitos. (Sozzo, 2000)

A su vez, surgió la implementación de TIC's tales como los circuitos cerrados de televisión (CCTV), alarmas, dispositivos biométricos, geolocalización, drones, botones de antipánico, etc., que vinieron a hacer de alguna manera, herramientas complementarias a las mencionadas. Al mismo tiempo, se posicionaron muy estrechamente a los servicios de vigilancia privada con las fuerzas de seguridad pública. La Prevención Situacional Ambiental fue una de las tácticas prevencionistas más utilizadas en materia de las perspectivas de seguridad ciudadana que incluyó a las nuevas tecnologías como una forma más innovadora para abordar los problemas de la inseguridad.

\section{Conformando una red panóptica}

Así se fueron diseñando políticas de implementación territorial donde la ciudadanía y las fuerzas participaban activamente en acciones para prevenir y reducir el delito.

Ahora bien, ¿de dónde nacieron algunas de estas ideas del control sistemático? A partir del sistema propuesto por Frederick W. Taylor las instituciones se transformaron en fábricas panópticas con el objetivo de mejorar la eficacia productiva y en beneficio económico. Las fábricas (hoy empresas) se convirtieron en instituciones que, a lo largo del tiempo, se fueron innovando con nuevos métodos de control y vigilancia.

La vigilancia en los centros laborales es especialmente efectiva porque es reflexiva, es decir, la gestión controla tanto a los trabajadores como a sí misma, y los estudios sobre la eficacia han tratado tanto de procesos administrativos y de supervisión como de los mecanismos productivos. (Herrera, 2006, p. 2).

Por ello, no sólo los Estados han creado nuevos métodos de control. Las empresas capitalistas del siglo XX han sido -y son actualmente- un lugar esencial para el ejercicio de la vigilancia laboral. Con la aplicación de las nuevas tecnologías de la información en los 
establecimientos laborales, los controles han aumentado e intensificado dentro de la gestión de la vigilancia disciplinaria y al mismo tiempo, estos métodos de vigilancia aparecieron también como alternativas para ser utilizados por los Estados modernos. (Herrera, 2006)

Pero la sociedad panóptica no sólo estaba formada por las instituciones privadas. Como mencionamos anteriormente, el Estado ha sido el principal actor, que con sus políticas no ha prohibido estas acciones, sino al contrario, las ha fomentado y empleado los mismos métodos de control social. También observamos cómo las TIC's convergieron en un único sistema, creando una gran malla de tecnología aplicada a la seguridad y vigilancia, ejerciendo un control social con los métodos anteriormente expuestos.

Para ello, las tecnologías aplicadas a la seguridad ciudadana están conformadas por los tres sistemas esenciales para el control que son: los sistemas de video-vigilancia públicos y privados, los sistemas de geolocalización, los sistemas biométricos y de controles accesos públicos.

A continuación, se indaga en torno a la conformación de esta red panóptica y se comienza a plantear la mirada sobre los sistemas de video-vigilancia que cuenta la Ciudad Autónoma de Buenos Aires y la Provincia de Buenos Aires. Al año 2013 se contabilizaban unas 1700 videocámaras domo, bajo el poder del Gobierno de la Ciudad Autónoma de Buenos Aires, que eran monitoreadas por la órbita de la Policía Metropolitana. (Ramallo, 2013)

En el 'Plan de Acción de Gobierno 2012- 2014' del Gobierno de la Ciudad Autónoma de Buenos Aires se expone que la política de seguridad se basa en gran medida, en tener controlado el espacio público con una amplia red en expansión de cámaras de seguridad.

Cuatro años atrás la Ciudad contaba con no más de 60 cámaras de seguridad. Hoy en día se está cerca de las 1.200 visualizadas y se planea llegar a fines del presente año 2011 a las 2.000 cámaras. Para los próximos años está previsto continuar con la extensión de la red, llegando a cubrir todos aquellos puntos estratégicos y sensibles de la Ciudad, de modo de llevar más seguridad y tranquilidad a los vecinos. (Gobierno de la Ciudad de Buenos Aires, 2011, pp. 12-13)

A esa cifra hay que sumarle, las cámaras controladas por la Policía Federal del Estado Nacional que también vigila el mismo territorio y posee una cantidad de 1200 videocámaras emplazadas en 300 puntos estratégicos de la Ciudad. Si le añadimos la cantidad de videocámaras de ambas fuerzas tenemos un total de 2900 videocámaras, que vigilan y controlan a los ciudadanos que habitan y transitan por la Ciudad Autónoma de Buenos Aires. (Ramallo, 2013).

La cantidad de videocámaras instaladas por parte de los gobiernos municipales en la Provincia de Buenos Aires, rondaría también las 9000 videocámaras. En la actualidad, en el conurbano hay en funcionamiento unas 6.156 cámaras y para fin de año se proyecta 
que lleguen a 8.000. En tanto, en toda la provincia, según datos del Ministerio de Seguridad, en la actualidad hay 9.000 cámaras activas y un total de 125 centrales de monitoreo, sobre un total de 135 municipios. (Gammacurta, 2013)

La red de videovigilancia pública y privada se complementan y trabajan en conjunto con los sistemas biométricos de control de acceso público, en su objetivo de tener controlado e implantar el orden dentro espacio público. De tal modo, los dispositivos de identificación como los DNI inteligentes, las tarjetas AFA PLUS, SUBE, Patentes vehiculares con chips (Diario La Voz, 2013), que constituyen en una herramienta eficiente para obtener los horarios de los recorridos, lugares frecuentados y asistidos de los individuos sujetos a esta nueva vigilancia invasiva. Si añadimos los sistemas de geolocalización, que a través de la conectividad de Internet, posibilitan que los dispositivos móviles y fijos conectados a la red, establezcan la ubicación casi exacta de los individuos sujetos al control y a la vigilancia.

Este control sistemático, se mantendrá informado constantemente con las cámaras del sistema de videovigilancia públicas y privadas de su jurisdicción, de la ubicación fija y/o móvil de los individuos a ser geolocalizados e identificados previamente por los dispositivos biométricos, donde la sociedad en su conjunto pasa a ser vigilada diaria y sistemáticamente durante todo el tiempo.

\section{Reflexiones finales}

A modo de conclusión, podemos decir que los sistemas (geolocalización, videovigilancia y biométricos) convergen en un único sistema de control y vigilancia, conformando así una gran red o malla panóptica, que concentra el poder y dominación de la información al servicio de los Estados. De esta manera, observamos cómo se generó una Mega Red Panóptica, la que, a través de la tecnología aplicada a la seguridad ciudadana, se encuentra en condiciones de vigilar y controlar a la sociedad en su conjunto.

Estas tecnologías aplicadas a la seguridad, dejan de lado los aspectos sociales generadores de conflictos, y se focalizan en conformar una red panóptica de manera obsecuente con las redes de control social desmedido y vulneración de derechos, confirmando que son tecnologías aplicadas a la seguridad ciudadana para generar nuevos métodos de control social sistemático. Podemos aseverar que el modelo benthamiano se lleva a cabo en su máxima expresión con la conformación del panoptismo tecnológico, creando una red panóptica que controla, tanto las conductas de los ciudadanos, como a las fuerzas de seguridad y sin dejar de lado sus mismos sistemas de seguridad propios al Estado, como una herramienta de autocontrol.

Dejando en evidencia que existen este tipo de organizaciones que utilizan a las TIC's para realizar tareas de inteligencia y sin estar vinculadas a la prevención en materia de 
seguridad, centrándose preferentemente en un control social generalizado. A su vez, crean un discurso desde la coyuntura política que determina acciones demagógicas y simplistas que pretenden solucionar las problemáticas sociales estructurales, recurriendo a la tecnología tal como si fuera la solución mágica para resolver las problemáticas sociales y culturales. Se comprende que es necesario y pertinente el uso de la tecnología aplicada a ámbito de la seguridad ciudadana pero con un uso responsable y medido, protegiendo todos los derechos y garantías constituciones. Entendiendo que la políticas caracterizadas por formular y ofrecer propuestas con ejes político-ideológico y preocupaciones sociales que resguardan los derechos y garantías de los ciudadanos son la más adecuadas, y comprendemos que la seguridad es una necesidad instituida por un Estado, donde las políticas públicas nacen dentro de un proyecto democrático en el cual la ciudadanía es un partícipe activo para prevenir delitos.

Al considerar que la justicia transicional es la encargada de dar soluciones como respuestas a las violaciones sistemáticas de los derechos humanos por parte de los Estados, vemos que la justicia transicional tendrá un nuevo desafío, como actor fundamental para intervenir y resguardar los derechos humanos en estos nuevos escenarios planteados por las implementaciones tecnológicas en materia de seguridad ciudadana. En este sentido, identificamos los riesgos que se presentan a la sociedad civil, la utilización desproporcionada de las TIC's por parte de algunas organizaciones estatales -que sin estar vinculadas a la presencia en materia de seguridad se convierten en una herramienta de control social explícito- con la consecuente pérdida de derechos por parte de los particulares.

Se propone articular medidas preventivas y de restauración con el espíritu de la justicia transicional, para no generar y provocar situaciones donde se vea la pérdida gradual de los derechos individuales de las personas, se limiten los derechos vinculados al anonimato, la privacidad, etc.

\section{Referencias bibliográficas}

Binder, A. (2009). El control de la criminalidad en una sociedad democrática. Ideas para una discusión conceptual, en Kessler, G. (Comp.), Seguridad y ciudadanía: nuevos paradigmas, reforma policial y políticas innovadoras. Buenos Aires: Edhasa.

Capistrán Bañuelos, J. I. (2003). Videovigilancia en la Sociedad Panóptica Contemporánea. Razón y palabra. Recuperado 11-09-2014. URL: http:/www.razonypalabra.org.mx

Castro, E. (2014). Introducción a Foucault. Buenos Aires: Siglo XXI.

Centro de Estudios Legales y Sociales (2003). Más derechos más Seguridad, Políticas públicas y seguridad en una sociedad democrática. Buenos Aires: CELS. 
Crawford, A. (1998). Crime Prevention and Community Safety. Politics, Policies and Practices, en Sozzo, M. (Comp.). Seguridad Urbana y Tácticas de Prevención del Delito, Cuadernos de Jurisprudencia y Doctrina Penal, $\mathrm{N}^{\circ} 10$. Buenos Aires: AdHoc.

Farhad Manjoo (2012). GPS para rastrear niños y perros. El control de los movimientos del núcleo familiar a través de la tecnología. El País, Nueva York. [En línea]. URL: http://tecnologia.elpais.com/tecnologia/2012/11/21/actualidad/1353507222_046236. html.

Foucault, M. (2002). Vigilar y castigar. Nacimiento de la prisión. Buenos Aires: Siglo $\mathrm{XXI}$.

Foucault, M. (2006). Seguridad, territorio y población. En Curso en el Collége de France: 1977-1978. Buenos Aires: Fondo de Cultura Económica.

Gammacurta, G. (2013). Es desparejo el uso de las cámaras en el conurbano, Ambito.com Recuperado 02-10-2014. URL: http://ambito.com/noticia.asp?id=706479

García Jiménez, R. (2009). El panoptismo: nuevas formas de control social. Revista Contribuciones a las Ciencias Sociales. Recuperado 18-08-2014. URL: http://www.eumed.net/rev/cccss/06/rgj2.htm

Gobierno de la Ciudad de Buenos Aires (2011). Plan de Acción de Gobierno 20122014. Recuperado 02-10-2014. URL: http://www.buenosaires.gob.ar/areas/hacienda/presupuesto2012/pdf/02_plan_de_a ccion_de_gobierno_2012_2014.pdf

González, P. (2003). Seguridad ciudadana. Cuaderno n². Guatemala: FLACSO. Herrera, C. G. (2006). El panóptico moderno. Revista de filosofía: A Parte Rei, № 46. La Voz (2013). La ITV costará \$150 y se controlarán autos por chips. LA VOZ. Recuperado 05-10-2014. UIRL: http://www.lavoz.com.ar/politica/la-itv-costara-150y-se-controlaran-autos-por-chip

López Martínez, D. L. (2013). Estado de la Industria de la Seguridad Privada en España, Tesis de Grado, Facultad de Ciencias Económicas y Empresariales, Universidad de León, España. URL: https://buleria.unileon.es/bitstream/handle/10612/2829/03117322V_GADE_julio13.p df.pdf?sequence=1. [En línea]

Mattelart, A. (2007). Sociedad del conocimiento, sociedad de la información, sociedad de control, Entrevista de Antonia García Castro a Armand Mattelart. Revista Cultures \& Conflits. Recuperado: 19-08-2014. URL: http://conflits.revues.org/2682. Ministerio de Seguridad de la Nación Argentina (2011). Nuevos vehículos, mejor seguridad en CABA. Recuperado 14-09- 
2014.URL:http://www.minseg.gob.ar/nuevos-veh\%C3\%ADculos-mejor-seguridaden-caba

Nievas, F. (1999). El control social de los cuerpos. Buenos Aires: Eudeba.

Oficina de Coordinación Nacional de Posicionamiento, Navegación, y Cronometría del gobierno de los Estados Unidos. Recuperado 08-09-2014. URL: http://www.gps.gov/systems/gps/spanish.php.

O`Malley, P. (2016). Riesgo, Neoliberalismo y Justicia Penal. Buenos Aires: Ad-Hoc.

Prince, A. y Jolías, L. (2011). Las TIC y su relación con la Seguridad Ciudadana: un marco de análisis a la problemática. Cuadernos de Seguridad No 14. Buenos Aires: Instituto Nacional de Estudios Estratégicos de la Seguridad, Ministerio de Seguridad, Presidencia de la Nación.

Ramallo, F. (2013). Porteños bajo el foco de las cámaras de vigilancia: cómo funciona el sistema de monitoreo. Recuperado 02-10-2014. URL: http://www.infotechnology.com/comunidad/Porteos-bajo-el-foco-de-las-camaras-devigilancia-como-funciona-el-sistema-de-monitoreo-20130826-0004.html

Rangugni, V. y Russo, D. (2010). La seguridad ciudadana. Cuadernos de Seguridad, $N^{\circ}$ 5. Buenos Aires: UNLa.

Sozzo, M. (2000). Seguridad Urbana y Tácticas de Prevención del Delito. Cuadernos de Jurisprudencia y Doctrina Penal, №10. Buenos Aires: Ad-Hoc.

Torrente Robles, D. (1997). La sociedad policial. Poder, trabajo y cultura en la organización local de la policía. Madrid: Centro de Investigaciones SociológicasUniversidad de Barcelona.

Varela, C. y Arriola, M. (2010). Comunidad y seguridad. Cuadernos de Seguridad, N 3. Buenos Aires: UNLa. 